\title{
Pour une typologie du résumé documentaire de type professionnel
}

\author{
Louise-L. Larivière \\ Université de Montréal et Université Concordia
}

Les deux types de résumés les plus connus sont, en français, la contraction de texte et, en anglais, l'abstract. Toutefois, il existe d'autres types de documents, utilisés à des fins professionnelles, rarement traités dans les ouvrages sur les résumés. Pour en tenir compte, il suffit d'élargir la notion de résumé pour y inclure tout document second obtenu par la réduction d'un document primaire. Cet article répertorie, donc, toutes les formes réduites d'analyse de contenu pour en arriver à caractériser les résumés documentaires de type professionnel. Pour ce faire, nous définissons, d'abord, le résumé documentaire (portant sur des documents) comme document second, comme phénomène de réduction et comme moyen de communication; puis, nous proposons une typologie des résumés documentaires à des fins professionnelles tout en ayant, au préalable, examiné d'autres typologies; finalement, nous définissons les résumés professionnels et énonçons les règles rédactionnelles sur lesquelles ils s'appuient. Par leur composante communicationnelle (finalité, destination, orientation, et par leurs autres caractéristiques (structuration, diversité), les résumés professionnels constituent de véritables documents susceptibles de former la population étudiante aux réalités du marché du travail. Aussi, ils devraient faire partie des programmes de formation des langagistes en lieu et place ou parallèlement aux résumés dits scolaires orientés uniquement vers une formation de l'esprit.

The best-known types of summaries are the academic "contraction de texte," in French, and the abstract, in English. However, there are numerous other types of summary documents that are used for professional purposes but are rarely dealt with in books on summarizing. This paper expands the notion of summary to include any secondary document obtained through the reduction of a primary document and proposes to characterize professional document summary types. The document summary is examined as a secondary 
document, as a reduction phenomenon, and as a means of communication. Existing summary typologies are reviewed, and an original typology for professional document summaries is proposed. Because they have real communicative contexts (purpose, audience, orientation) and distinct characteristics (structure and diversity), professional summaries constitute real documents that can be used to prepare students to write for the workplace. Consequently, they have a role in courses that train language practitioners and need not be restricted to school exercises designed only to develop general intellectual skills.

\section{Introduction}

Les deux types de résumés les plus connus sont, en français, la contraction de texte et, en anglais, l'abstract. Le premier type, la contraction de texte, est un résumé surtout utilisé à des fins scolaires et consiste en une réduction à l'échelle du texte de départ. En France, ce résumé constitue, bien souvent, une épreuve à subir pour être reçu à des concours d'entrée ou pour obtenir un diplôme (ex. l'épreuve du résumé au baccalauréat). Il est, de plus, régi par des normes qui sont définies dans le Bulletin officiel de l'Éducation nationale du 7 juillet 1983 et reproduites dans Sabbah 1991 :9 (voir Annexe I). Par conséquent, plusieurs ouvrages sur le résumé sont conçus de façon à satisfaire à ces normes dont Arambourou 1991, Carloni 1984, Fournier/Dutertre 1993, Giquel 1990, Maury 1982, Moreau 1984, Sabbah 1991 et Stalloni 1990. Au Québec, certains articles ont porté sur des moyens pratiques d'améliorer la qualité des résumés (Larivière 1989, Meney 1993) ; un seul ouvrage en français (Valentine1990) porte sur la contraction de texte et s'adresse principalement à des langagistes, soit à ceux et à celles qui exercent des activités professionnelles reliées au traitement de la langue (rédaction, révision, terminologie, traduction).

Le deuxième type, l'abstract, traduit en français par «résumé analytique» (ISO 1981, Université Laval 1983), "résumé» (AFNOR 1984) ou "analyse» (ISO 1976), est un résumé professionnel peu traité, en français, en dehors des ouvrages des spécialistes du domaine de la bibliothéconomie et des sciences de l'information (voir, entre autres, Guinchat/Menou 1990). Il consiste en un résumé qui rend compte du contenu d'un document, soit de façon signalétique en n'en donnant que les grands points de développement à la manière d'une table des matières (résumé indicatif), soit de façon analytique en donnant l'aboutissement de la recherche, i.e. les résultats et les conclusions (résumé informatif). Les résumés indicatifs et informatifs ont pour but de renseigner les usagers et les usagères sur la pertinence du document consulté en vue d'un travail de recherche donné. 
Il existe, toutefois, d'autres types de documents, utilisés à des fins professionnelles dans certains secteurs d'activité, qui sont rarement traités dans les ouvrages sur les résumés parce qu'ils ne sont, sans doute, pas considérés comme tels, bien qu'ils le soient. Nommons, entre autres, la quatrième de couverture (domaine de l'édition), le résumé de rapport (domaine de la gestion), le résumé d'arrêt (domaine du droit) et le «chapeau» (domaine du journalisme). Aussi, nous nous proposons, dans cet article, d'étendre la notion de résumé en répertoriant toutes les formes réduites d'analyse de contenu pour en arriver à caractériser les résumés documentaires à des fins professionnelles.

Pour ce faire, nous définirons, d'abord, le résumé documentaire d'un triple point de vue : comme document second, comme phénomène de réduction et comme moyen de communication ; puis, nous proposerons une typologie des résumés documentaires à des fins professionnelles à partir de critères bien définis tout en ayant, au préalable, examiné d'autres typologies ; finalement, nous définirons les résumés professionnels et énoncerons les règles rédactionnelles sur lesquelles ils s'appuient.

\section{La nature du résumé documentaire}

Pour préciser la nature du résumé documentaire, il faut envisager la place qu'occupe ce dernier parmi les documents seconds, les phénomènes de réduction et les moyens de communication.

\subsection{Un document second}

Un résumé est, avant tout, une analyse de contenu. À ce titre, il est un document second, en ce sens qu'il est le produit d'une transformation d'un document original, appelé document premier ou primaire, au même titre que d'autres documents mais de façon différente. Il existe, en effet, plusieurs documents seconds issus de divers procédés de transformation :1) ceux dont le contenu ou message est le même que celui du document premier, soit les documents de substitution dont la paraphrase, la parodie et l'adaptation qui se présentent dans la même langue que celle du document premier, soit la traduction qui se présente dans une langue autre que celle du document premier, soit l'illustration qui se présente comme un équivalent non-textuel d'un document premier textuel ;2) ceux dont le contenu va au delà de celui du document premier, soit les documents d'adjonction dont le compte rendu critique d'un document, l'explication ou le commentaire de texte ; et3) ceux dont le contenu est en deça du document premier, soit les documents de réduction dont le résumé qui peut être dans la même langue que celle du document premier (résumé intralinguistique) ou être dans une langue autre (résumé interlinguistique). Ces documents sont regroupés dans le Tableau 1 : Analyse de contenu - Procédés de transformation et produits dérivés. 


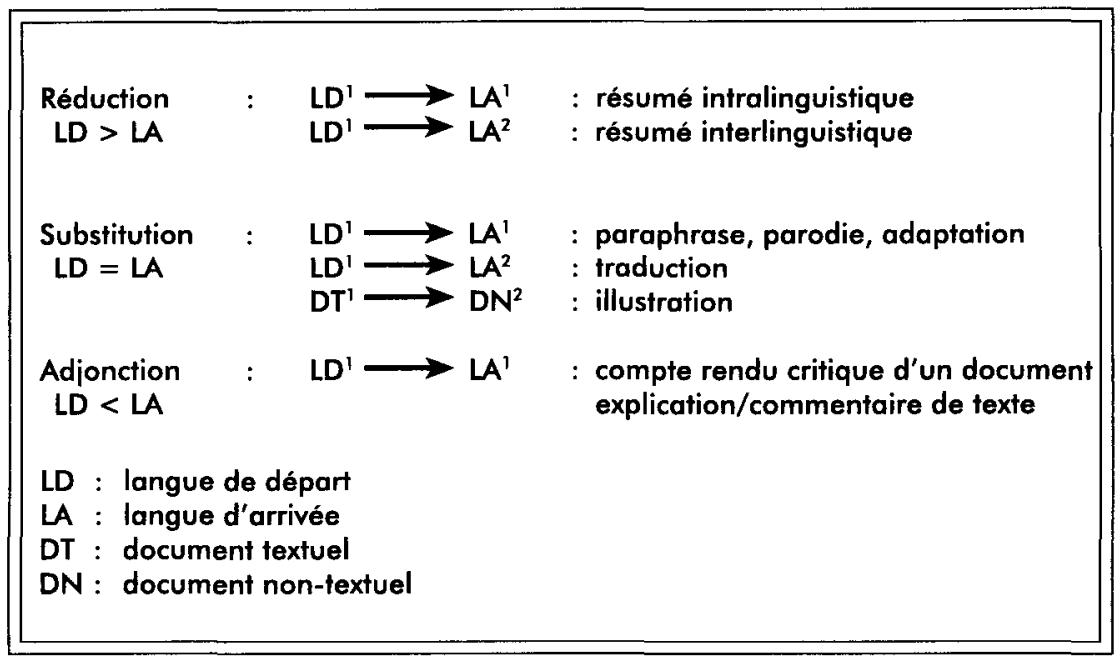

Tableau 1 : Analyse de contenu

\section{Procédés de transformation et produits dérivés}

À ce stade-ci, posons comme première définition du résumé documentaire qu'il est un document second obtenu par la réduction d'un document primaire.

\subsection{Un phénomène de réduction}

Le résumé est un phénomène de réduction linguistique, mais il n'est pas le seul. Ce phénomène qui consiste à réduire (diminuer) l'étendue d'une forme linguistique cible par rapport à celle d'une forme source tout en conservant une partie du contenu de cette dernière, se situe tant au plan de la langue (réduction grammaticale) qu'à celui des documents (réduction documentaire). Au plan grammatical, la réduction se manifeste à tous les échelons de la chaîne parlée : celui des morphèmes (ajouter -> ajout), des mots (article $->$ art.), des syntagmes (machine calculatrice $->$ calculatrice) et des phrases (J'ai une robe + Elle est bleue -> J'ai une robe bleue). Comme cette forme de réduction ne touche pas directement à notre propos, nous n'en traiterons pas davantage. Nous nous attarderons plutôt à la réduction documentaire.

La réduction documentaire, soit le processus par lequel on obtient un document réduit, porte soit sur des manifestations, soit sur des documents primaires. Lorsque la réduction porte sur des manifestations, ces dernières peuvent consister en des discussions (audience ou réunion) - et se rendre au moyen d'un compte rendu ou d'un procès-verbal de séance —, ou en des actes (événement, activité, mission) — et se rendre au moyen d'un compte rendu (de faits) ou d'un rapport. 
Lorsque la réduction porte sur des documents, plusieurs critères sont en jeu :la nature et le nombre des documents de départ (documents primaires) et la nature du document d'arrivée (forme du résumé). De quels documents de départ s'agit-il? De documents écrits ou de documents audio-visuels? La réduction porte-t-elle sur un ou plusieurs textes? Quelle forme prend alors le produit réduit? Une forme signalétique (titre, mot-clé, table des matières, index) ou une forme narrative? Si le document réduit porte sur des documents écrits, on parlera de résumé textuel, s'il porte sur des documents audio-visuels, on parlera de synopsis. $\mathrm{Si}$, au départ, il n'y a qu'un texte à résumer, on parlera de résumé de texte comme produit réduit ; si, au départ, il y a plus d'un texte à résumer, on parlera de synthèse de textes comme produit réduit. Si le produit réduit adopte une forme signalétique plutôt que narrative, il relèvera plus de ce type de réduction documentaire que l'on appelle «indexation» que du résumé (voir le Tableau 2 : Phénomène de réduction documentairà.

À la lumière des explications fournies sur le résumé comme phénomène de réduction, reformulons la définition du résumé documentaire comme suit :Document second qui reproduit, sous forme de document réduit narratif, le contenu soit d'un seul

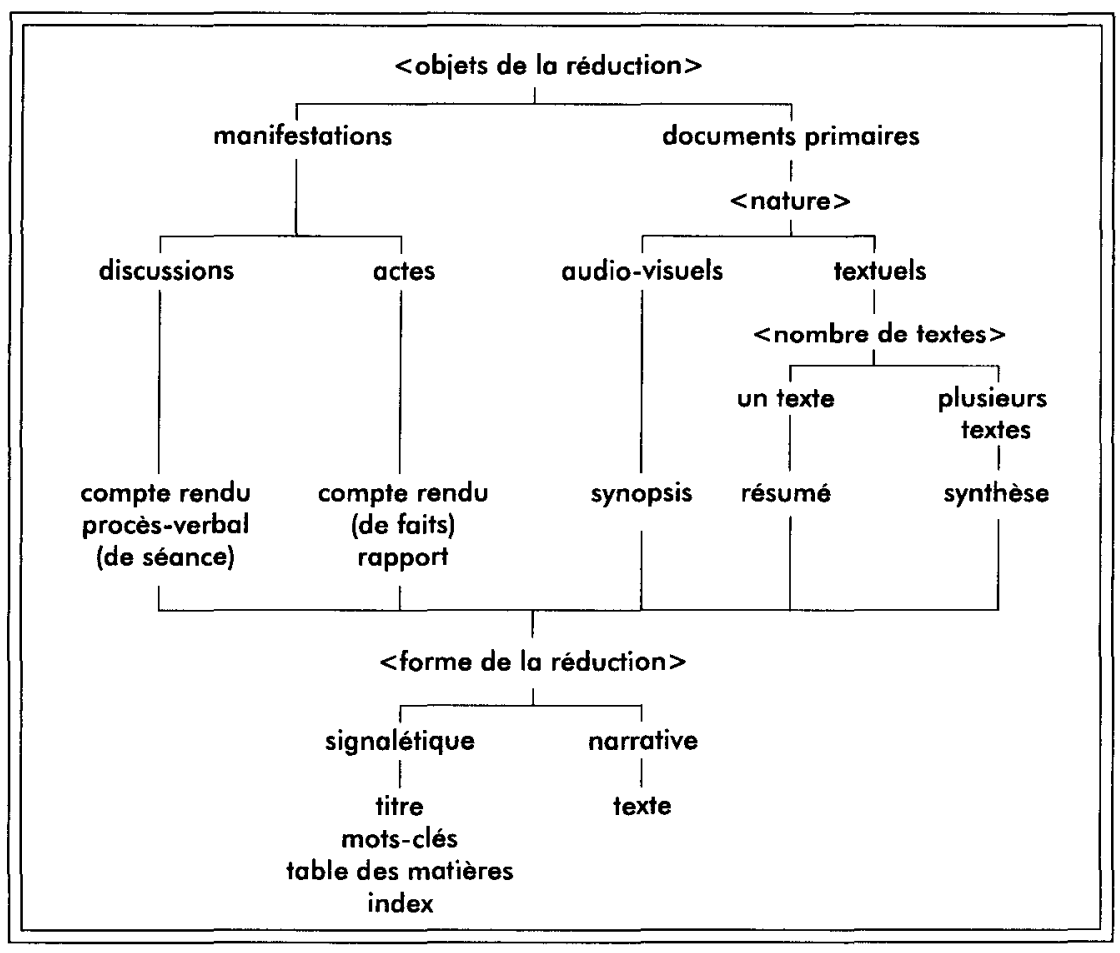

Tableau 2 : Phénomène de réduction documentaire 
texte primaire (résumé de texte), soit de plusieurs textes primaires (synthèse de textes). Plus haut, nous avons parlé de "résumé textuel» et de "résumé de texte», termes qui ne sont toutefois pas synonymes. En effet, le terme «résumé textuel» signifie que le produit provient de la réduction d'un ou de plusieurs TEXTE(S) DE DEPART, tandis que le terme "résumé de texte» signifie le PRODUIT LUI-MÊME de la réduction d'un seul texte de départ. Dans la pratique, toutefois, il ne risque pas d'y avoir de confusion puisque, généralement, c'est du produit dont on parlera. Ainsi en parlant de "résumé de texte», on comprendra qu'il s'agit de la réduction d'un seul texte de départ et en parlant de "synthèse de textes», on comprendra qu'il s'agit de la réduction de plusieurs textes de départ.

\subsection{Un moyen de communication}

Dans l'introduction, nous avons parlé de deux types de résumés les plus courants, soit la contraction de texte comme résumé scolaire et le résumé analytique comme résumé professionnel. Or, selon Bernié (1993 :3), le résumé devrait comprendre "une composante communicationnelle, incluant l'ensemble des contraintes imposées par la situation dans laquelle prend place l'activité résumante : pour qui et pour quoi résume-t-on?». En supposant que cette composante soit nécessaire pour considérer les résumés comme des documents, on peut alors se demander si les deux types de résumés mentionnés comportent cette composante. Sans entrer dans toute la dialectique de Bernié, disons que, pour lui, l'activité résumante «bête et méchante» (35) du système scolaire n'inclut pas les contraintes mentionnées plus haut. Le résumé scolaire, selon les ouvrages traditionnels, a pour finalité d'être un exercice d'intelligence, de jugement et de style, finalité qui ne répond à aucune intention de communication : informer, exprimer une opinion, inciter à l'action ou divertir. Il n'est donc qu'une activité de simulation (35). De plus ce résumé, toujours selon Bernié, n'a pas de destinataires autres que les correcteurs et les correctrices qui ne sont pas, cependant, de véritables destinataires. En effet, le résumé n'ayant pas été conçu à leur intention, ils et elles ne peuvent y réagir qu'en coulant l'élève qui n'a pas rédigé son résumé conformément aux règles établies.

Quant aux résumés analytiques dont nous avons parlé, ils ont une finalité bien définie - informer - et des destinataires bien identifiés - des chercheurs et des chercheuses. Seuls ces résumés satisfont à une situation de communication. Aussi, si nous voulons considérer le résumé comme un genre à part entière, soit un type de document satisfaisant à un acte de communication, il faut éliminer le résumé scolaire de notre typologie du résumé et ne considérer que les résumés qui répondent à un acte de communication. Aussi, nous ne considérerons que les résumés professionnels qui ont une finalité propre et des destinataires définis. Par conséquent, la contraction 
de texte, comme forme de résumé scolaire, sera exclue de notre typologie mais non la contraction de texte utilisée à des fins professionnelles (par exemple, les condensés rédigés par des langagistes résumant du courrier adressé à des ministres).

\section{La typologie du résumé documentaire}

\subsection{Les typologies existantes}

Il existe certaines typologies du résumé. Pouzet (1981 :18) propose une typologie de la contraction de texte à partir des contraintes imposées par le Bulletin officiel de l'Éducation nationale dont nous avons parlé plus haut et applique sa grille à divers types de résumés (voir Annexe II).

Russell (1994) propose une typologie plus générale. Elle s'attarde, d'abord, à classer les résumés en fonction du critère pratique de la finalité (38). Aussi, elle distingue le précis (contraction de texte) ou school-sponsored summary (résumé scolaire) qui consiste en un exercice dont le but est de développer des aptitudes rédactionnelles; l'abstract (résumé analytique) qui donne un aperçu du contenu d'un document à des fins de recherche ; lesummary record qui condense un discours oral ; lesminutes (procès-verbal de séance) qui consigne ce qui a été dit lors de réunions ; le executive summary (résumé de rapport) qui introduit un document d'affaires auquel il est joint ; les popularized summaries qui consistent en des condensés ou des adaptations destinés à un vaste public ; lesheadlines (manchettes), leads (chapeaux), press releases (communiqués) ainsi que les articles eux-mêmes relatant un événement, procédés qui sont tous utilisés dans le domaine du journalisme.

Russell adopte, ensuite, une approche plus théorique. Elle passe en revue diverses études qui s'appuient sur quatre types de considérations : structurales, métatextuelles, cognitives et contextuelles. Sur le plan structural (38-40), Russell démontre la nécessité d'établir un lien entre la structure du texte de départ (TD) et celle du texte d'arrivée (TA), i.e. le résumé, lien devant servir de critère de classification des résumés. Le type de résumé qui reflète le mieux ce lien est le résumé analytique d'une étude expérimentale qui adopte la même structure que le TD : objet, but, méthode, résultats et conclusions. Beaudet (1994) dans un article qui, en dépit du titre, ne présente pas une typologie des résumés fonctionnels, a décrit des modèles structuraux de textes de différents types (descriptif, narratif, expositif, argumentatif et instructif) devant servir à classifier les résumés.

Sur le plan métatextuel (40-42), Russell fait état d'études qui ont établi des liens extérieurs entre le TD et le TA. Ainsi, elle invoque le critère de la distanciation pour indiquer le rôle que joue l'analyste ou résumiste (rôle de substitution de l'auteurE ou d'intermédiaire entre l'auteurE et la ou le destinataire), le critère de la structure des 
phrases pour distinguer, entre autres, le résumé indicatif du résumé informatif (ou descriptif), le critère de la linéarité pour distinguer le résumé de texte de la synthèse de texte, le critère de la formulation du TA dans des termes autres ou dans les mêmes termes que ceux du TD pour distinguer les résumés scolaires des résumés professionnels, les condensés et les procès-verbaux des résumés analytiques.

Les stratégies de contraction de texte servent, par ailleurs, de critères pour distinguer les types de résumés sur le plan cognitif (43). Russell fait part, alors, d'études qui distinguent entre les résumés mature et les résumés immature selon le degré de profondeur de l'analyse du TD.

Finalement, Russell évoque cinq critères de nature contextuelle (44-45) reliés à la situation de communication :1) le critère de la cooccurrence des TD et des TA permet de distinguer le résumé prospectif, ex. la proposition de communication (conference paper abstract) et le résumé rétrospectif, ex. le résumé analytique d'un répertoire documentaire (abstract in an abstracting or reference journal), qui apparaissent tous deux sans le TD, du résumé d'article (abstract of a research article) qui, lui, apparaît en même temps que le TD ;2) le critère de la personne émettrice conduit soit à un résumé d'auteurE (author abstract), soit à un résumé d'analyste (access abstract) ;3) le critère du but poursuivi conduit à un résumé qui fournit les grandes lignes d'un document (résumé indicatif) ou à un résumé qui traite du contenu en profondeur (résumé informatif) ;4) le critère du type de public visé conduit à un résumé pour spécialistes ou à un résumé grand public ;5) le critère du médium utilisé : ex. les discours oraux donnent lieu à des résumés écrits dont le procès-verbal \$) le critère des divers domaines d'activités conduisent à autant de résumés.

\subsection{Notre proposition de typologie}

Notre analyse du phénomène de la réduction documentaire (voir plus haut) nous a amenée à distinguer divers types de résumés documentaires : ceux qui traitent de manifestations et ceux qui traitent de documents. Parmi ces derniers, il y a ceux qui portent sur des documents audio-visuels et ceux qui portent sur des documents écrits. Ces résumés textuels comprennent les résumés de texte qui portent sur un seul document et les synthèses de textes qui portent sur plusieurs documents. Nous nous attarderons à ne classifier que les résumés de texte.

Le résumés de texte s'appuient sur plusieurs critères de classification. Le premier est le choix du contenu du document à résumer. S'agit-il de résumer le document dans son entièreté ou de ne faire part que de certaines composantes ou aspects de ce document? Une analyse intégrale (et linéaire) du document donnera lieu à une contraction de texte, tandis qu'une analyse sélective donnera lieu à des résumés sélectifs. 
Le deuxième critère est l'intention de communication. Quel est le but poursuivi par le résumé sélectif, informer ou promouvoir? Pour l'un et l'autre but, un troisième critère s'applique (cooccurrence). Le résumé apparaît-il concurremment ou non avec le TD? Que la réponse soit oui ou non, il faut faire intervenir un quatrième critère qui est celui du domaine. À quel domaine du savoir appartient le résumé?

Les résumés qui ont pour but d'informer et qui apparaissent en même temps que le TD se situent dans le domaine de la gestion et dans le domaine des sciences. Pour ce qui est du domaine de la gestion, un cinquième critère s'applique : l'orientation du résumé. Le résumé annonce-t-il un document à venir (orientation prospective) ou rappelle-t-il un document déjà paru (orientation rétrospective)? Par exemple, le résumé de gestion à caractère prospectif annonce un rapport ou une étude. Comme résumés de gestion à caractère rétrospectif, citons le résumé des conclusions partielles comme celui que l'on trouve dans la conclusion générale d'un rapport et les résumés d'enquête et de questionnaire (Jewinski/Jewinski 1992). Pour ce qui est des résumés scientifiques, citons le résumé d'article de revue savante.

Les résumés qui ont pour but d'informer, mais qui n'apparaissent pas en même temps que le TD, se situent dans trois domaines : domaine de la documentation, domaine du droit et domaine des sciences. Pour ce qui est des sciences, le cinquième critère s'applique (orientation du résumé). Le résumé scientifique à caractère prospectif porte sur la proposition de communication (pour une conférence), tandis que celui à caractère rétrospectif porte sur le résumé analytique d'une étude expérimentale qui s'apparente aux résumés informatifs que l'on retrouve dans les répertoires analytiques et les bases de données documentaires. En documentation, citons l'annotation, le résumé indicatif et le résumé informatif, tandis qu'en droit, citons le résumé d'arrêt (comme celui que l'on retrouve, par exemple, dans Jurisprudence Express).

Pour ce qui est des résumés qui ont pour but de promouvoir et qui, à titre d'outils de promotion ont un caractère plus subjectif, nous les retrouvons dans deux domaines, celui du journalisme (le «chapeau» qui coiffe un article de revue grand public ou de journal) et celui de l'édition (la quatrième de couverture et l'argumentaire).

Ces divers résumés de texte sont schématisés sous forme arborescente dans le Tableau 3 : Typologie des résumés de texte

Tout le phénomène de réduction documentaire s'appuie donc sur les critères de classification suivants regroupés autour de trois paramètres : les objets de la réduction, les documents primaires de départ et les documents d'arrivée :

- Objets de la réduction

- nature (manifestations ou documents primaires) 


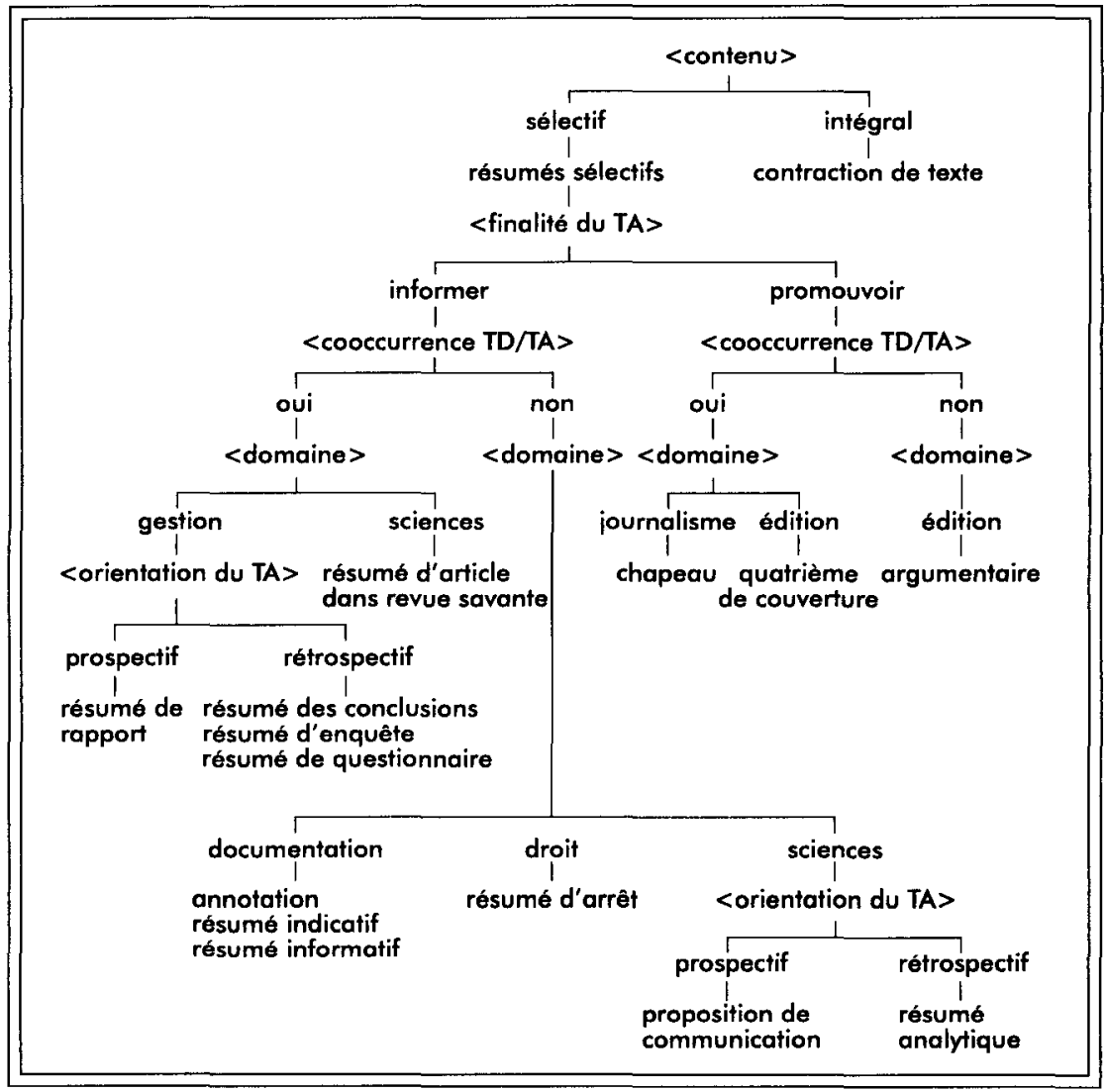

Tableau 3 : Typologie des résumés de texte

- Documents primaires de départ (DD)

- nature (documents textuels ou documents non-textuels)

- nombre de documents textuels (un ou plus d'un document)

- contenu à conserver (contenu sélectif ou intégral)

- Documents d'arrivée ou résumés (DA)

- forme (narrative ou signalétique)

- finalité (informer ou promouvoir)

- cooccurrence (apparaît ou non avec le DD)

- domaine d'appartenance (gestion, sciences, documentation, droit, journalisme, édition)

- orientation (prospective ou rétrospective) 
Nous n'avons pas tenu compte du critère de linéarité (déroulement du résumé = déroulement du texte), ni de celui de la distanciation linguistique entre le document d'arrivée et le document de départ. Ces critères ne sont pas pertinents pour les types de documents décrits. Dans notre typologie, nous n'avons pas, non plus, tenu compte (bien que nous aurions pu le faire) de la personne émettrice du résumé : auteurE ou analyste. Disons toutefois que :

1) Certains résumés ne sont jamais rédigés par les auteurEs des documents primaires mais par des analystes : annotation, résumé indicatif, résumé informatif (documentation), résumé d'arrêt (droit) ;

2) Certains résumés peuvent être rédigés par les auteurEs ou par une équipe éditoriale : chapeau (journalisme), quatrième de couverture et argumentaire (édition) ;

3) Certains résumés sont habituellement rédigés par les auteurEs : résumé d'article de revue savante ;

4) Certains résumés sont toujours rédigés par les auteurEs : proposition de communication (pour une conférence) ainsi que le résumé de rapport et le résumé des conclusions (gestion).

\section{Les résumés professionnels ${ }^{1}$}

Après avoir établi une typologie des résumés professionnels, qui sont des résumés de type sélectif, nous allons maintenant préciser la nature de chacun de ces résumés et exposer, bien que brièvement, les normes rédactionnelles sur lesquelles ils s'appuient le cas échéant. Pour ce faire, nous avons regroupé les résumés selon le critère de la cooccurrence, soit la publication concurrente ou non du résumé et du texte de départ à l'intérieur d'un même document.

\subsection{Résumé en cooccurrence avec le texte de départ}

Dans ce groupe de résumés, nous trouvons ceux qui ont pour but d'informer un public lecteur sur le texte de départ et qui se retrouvent dans le domaine de la gestion et dans le domaine scientifique, de même que ceux qui ont pour but de promouvoir, auprès du public lecteur, le texte de départ et qui se retrouvent dans les domaines du journalisme et de l'édition. 
Dans le domaine de la gestion, le résumé de rapport (executive summary) est le plus connu ; il est largement décrit dans les ouvrages de rédaction professionnelle, entre autres Blicq (1987), Larose (1992), Robinson (1985). Ce résumé consiste en un condensé prospectif et non-technique des points saillants d'un rapport, rédigé à l'intention des gestionnaires et placé entre la page de titre et la table des matières du rapport. En anglais, il se nomme justement executive summary parce qu'il s'adresse à des destinataires qui ne sont pas nécessairement des spécialistes d'un domaine, mais qui auront à appliquer les mesures préconisées dans le rapport sans avoir à en lire tout le développement. Il se compose de trois sections :1) l'objet du rapport ;2) les résultats et les conclusions ;3) les recommandations. L'objet ne donne qu'un aperçu du problème soulevé dans le rapport, mais suffisant pour en faire comprendre les conclusions et les recommandations qui suivent.

Parmi les résumés de gestion rétrospectifs, signalons le résumé des conclusions d'un rapport, le résumé d'enquête et le résumé de questionnaire. Pour ces deux derniers, nous renvoyons à l'étude de Jewinsky/Jewinsky (1992). Le résumé des conclusions se situe dans la première partie de la conclusion générale d'un rapport et énumère les conclusions partielles auxquelles ont abouti les points de développement du rapport afin de préparer le public lecteur à mieux comprendre les recommandations qui seront faites dans la deuxième partie de la conclusion générale. Ce résumé n'est pas soumis à des règles de contenu particulières. Il n'est soumis qu'à des règles de style (cohésion, concision, exhaustivité) propres à tout document professionnel.

Dans le domaine scientifique, nous retrouvons le résumé d'article de revue savante. Ce résumé, placé au début de l'article et rédigé généralement par l'auteurE, ne répond pas à des normes strictes. Il se veut tantôt de nature indicative, tantôt de nature informative (ce qui est le plus généralement le cas). Dans le présent numéro de Technostyle, Kjersti Fløttum souligne la nature argumentative de ce type de résumé qu'elle nomme "résumé scientifique».

Les deux types de résumés promotionnels qui suivent sont largement utilisés bien qu'ils ne soient pas soumis à des régles strictes d'écriture. Dans le domaine de l'édition, la quatrième de couverture (back cover) est un document de promotion qui, comme son nom l'indique, se situe sur la quatrième page couverture d'un livre et qui fournit des indications sommaires sur unE auteurE et son ouvrage. On y retrouve, en général, certains des éléments suivants : un aperçu descriptif et critique (toujours positif, il va sans dire) du contenu de l'ouvrage, une brève notice biographique de l'auteurE, des notes sur la collection, des extraits de l'ouvrage (préface ou corps du texte). Certaines maisons d'édition consultées disent ne pas avoir de normes précises quant au contenu et à la rédaction de ce type de résumé qui pourrait constituer un domaine de recherche intéressant. 
Dans le domaine du journalisme, le chapeau (introductory paragraph), situé entre le titre et le texte d'un article de journal ou de magazine, donne, de façon percutante, l'idée marquante du texte pour attirer l'attention du public lecteur sur ce dernier et l'inciter à le lire. Selon certains magazines consultés, ce document est rarement rédigé par l'auteurE et n'est pas soumis à des normes d'écriture. Ce document pourrait, également, constituer un domaine de recherche intéressant.

\subsection{Résumé non en cooccurrence avec le texte de départ}

Les résumés qui ne sont pas en cooccurrence avec le texte de départ se situent dans les domaines de la documentation, du droit, des sciences et de l'édition et répondent, pour la plupart, à des protocoles stricts d'écriture.

En documentation, nous retrouvons l'annotation, le résumé indicatif et le résumé informatif. L'annotation (annotation) consiste en un «[b]ref commentaire ou explication d'un document ou de son contenu, ou même une très brève description, habituellement ajoutée en note après la référence bibliographique du document» (ISO 1976 :37, ISO 1981 :18) dans les répertoires documentaires, les listes de lectures ou les bibliographies (Landau 1966 :17). Il peut servir soit à préciser ou à compléter un titre non-significatif ou trompeur, soit à attirer l'attention du public lecteur sur des éléments pertinents entourant le document (identité des auteurEs, buts et limites, orientation idéologique, liens avec d'autres ouvrages, appréciation [ex. À lire absolument], public visé, etc.)

Le résumé indicatif ou résumé signalétique (indicative abstract) est un type de résumé sélectif utilisé à des fins documentaires qui se contente de signaler ou d'énumérer, de façon extérieure et brève, l'objet et les grandes lignes contenus dans le document primaire à la manière d'une table des matières. C'est donc un regard extérieur, impartial et objectif, d'un ou d'une analyste qui nous indique «le genre du document, les principaux sujets couverts et la manière dont les faits sont traités» (ISO 1981 :17).

Le résumé informatif ou résumé analytique (informative abstract) est un type de résumé sélectif utilisé à des fins documentaires qui est «une représentation abrégée du document renseignant sur les informations quantitatives ou qualitatives apportées par l'auteur» (AFNOR 1984 :6, ISO 1981 :17). Il porte sur une étude ou sur une recherche en en explicitant l'objet, la méthode, les résultats et les conclusions afin d'informer sur une question donnée. En bref, il énonce ce que le document dit ou, en d'autres termes, c'est le texte qui révèle lui-même son contenu : c'est donc undocument vu de l'intérieur qui discute de la recherche et non de l'article qui décrit la recherche. Notons que le terme "résumé analytique» est utilisé, en français, à la fois pour traduire le générique «abstract» et comme synonyme du spécifique "résumé informatif», ce qui peut porter à confusion. 
Ces trois types de résumés documentaires comportent des règles d'écriture, décrites surtout dans les normes internationales et nationales, de même que dans les manuels de rédaction professionnelle rédigés en anglais. Comme nous le mentionnions dans l'introduction, peu d'ouvrages rédigés en français traitent de ce résumé.

En droit, le résumé d'arrêt (summary of a judgment) est un résumé sélectif qui relève les éléments essentiels d'un arrêt de cour (décision ou jugement) : autorité de l'arrêt, histoire des procédures, faits, question en litige, argumentation (ratio decidendi), dispositif, dissidence. La Société québécoise d'information juridique a établi des règles d'écriture (SOQUIJ 1993) qu'elle applique dans son recueil d'arrêts Jurisprudence Express. Sur le plan du contenu, chaque arrêt se compose des quatre parties suivantes :1) la manchette (titre ou mots-clés);2) l'identification du litige (énoncé des procédures entreprises et issues recherchées et obtenues) ;3) le contexte (présentation des faits, des parties en cause et des relations entre elles, des admissions, documents ou dispositions législatives nécessaires au litige et des prétentions des parties sur lesquelles la magistrature s'est prononcée) ;4) la décision (points discutés par la ou le juge) ; et se termine par l'identification de la cause.

Dans le domaine des sciences, nous retrouvons la proposition de communication (paper abstract) qui consiste en un document prospectif qui décrit un projet à présenter, sous forme de communication, lors d'un colloque ou d'un congrès. Bien qu'il n'y ait pas de règles écrites sur la rédaction de ce document, il est d'usage d'y utiliser les éléments suivants :1) énoncé de l'hypothèse à démontrer ;2) description de la démarche utilisée pour effectuer la recherche ;3) aperçu des résultats obtenus ou envisagés. Nous retrouvons, également, le résumé analytique qui est un document rétrospectif qui porte sur une étude expérimentale, qui s'apparente aux résumés documentaires de type informatif et qui pourrait servir à des fins diverses.

Dans le domaine de l'édition, l'argumentaire (blurb) qui consiste en un article faisant partie d'un catalogue annonçant la parution d'un ouvrage, s'apparente, la plupart du temps, à la quatrième de couverture du livre annoncé. Pas plus que pour ce dernier document, il ne repond à des critères stricts d'écriture si ce n'est l'utilisation d'un ton laudatif.

Etant donné que le résumé de texte s'enseigne dans certaines écoles de traduction au Canada, il nous a semblé tout à fait approprié de s'interroger sur la nature des résumés que l'on enseigne dans ce secteur d'études particulier. Comme ce dernier ne touche pas, toutefois, tout le lectorat de Technostyle, nous avons reporté le résultat de nos recherches en annexe. (Voir l'Annexe III, Description des cours de réduction de textes offerts dans les écoles de traduction au Canada, qui reproduit la description des cours sur le résumé qui apparaît dans les annuaires de cinq de ces écoles où s'enseigne le résumé. Ce tableau s'accompagne d'un commentaire.) 


\section{Conclusion}

Dans cet article, nous avons distingué les résumés scolaires des résumés professionnels qui sont les seuls à répondre à un véritable processus de communication. Parmi ces derniers, nous nous sommes attardée à établir une typologie de ceux qui portaient sur des documents et plus particulièrement sur des documents textuels en fonction de critères bien déterminés. De plus, nous avons, brièvement, défini les résumés professionnels et exposé les normes rédactionnelles sur lesquelles ils s'appuient.

De par leur composante communicationnelle, les résumés professionnels constituent de véritables documents susceptibles de former la population étudiante aux réalités du marché du travail. Leurs caractéristiques sont, en effet, les suivantes :1) ils ont une finalité puisqu'ils sont conçus dans un but précis : informer ou promouvoir 2) ils ont une destination puisqu'ils sont conçus en fonction de vrais destinataires $\mathfrak{3}$ ) ils constituent un document réel puisqu'ils sont orientés vers une pratique professionnelle ;4) certains d'entre eux respectent un protocole d'écriture strict (résumé de rapport, annotation, résumé indicatif, résumé informatif, résumé d'arrêt et résumé analytique) ;5) ils préparent directement au marché du travail ;6) ils ont une grande diversité de formes ; 7) leur caractère sélectif exige une plus grande capacité de synthèse.

Par conséquent, ces résumés professionnels devraient faire partie des programmes de formation des langagistes en lieu et place des résumés dits scolaires qui sont orientés uniquement vers une formation de l'esprit ou parallèlement à eux. Nous croyons que l'enseignement de ces résumés professionnels, tout en préparant les étudiants et les étudiantes en traduction/rédaction au milieu du travail, pourront tout aussi bien «développer leur intelligence, leur jugement que leur style».

\section{NOTES}

1 Nous tenons à remercier les personnes suivantes qui nous ont fourni de la documentation ou de l'information sur leurs pratiques professionnelles : Me Monique Desrosiers et Me Micheline Montpetit (Société québécoise d'information juridique), M. Patrick Coppens (Services documentaires multimédia inc.), Mme Paule Beaugrand-Champagne (autrefois du magazine L'Actualité), Mme Jannick Belleau et M. Paul Simard (Régie du cinéma du Québec), Mme Anik Charrette (Tv-Hebdo). M. André Vanasse (Éditions XYZ), M. Alain Stanké (Éditions Alain Stanké), Mme Monique Desrochers (autrefois de l'École de bibliothéconomie et des sciences de l'information de l'Université de Montréal) et Mme Marielle Saint-Amour (Faculté de l'éducation permanente de l'Université de Montréal). 


\section{ANNEXE I}

\section{Définition officielle}

Voici la définition officielle de l'exercice :

Le résumé suit le fil du développement. Il donne du texte, dans le même ordre, une version condensée mais fidele. Il ne change pas le système de l'énonciation :il reformule le discours du texte initial sans prendre de distance (c'est-à-dire en s'abstenant d'indications telles que «I'auteur déclare que... montre que...»). Il s'interdit un montage de citations : le candidat exprime dans son propre langage les assertions du texte. Il peut cependant, lorsqu'il s'agit de mots clés, qu'il serait absurde de remplacer par de mauvais équivalents, reprendre les mots du texte et, par exception, citer entre guillemets une formule particulièrement significative.

Pour que ce travail ait tout son sens et soit conduit avec rigueur, il est nécessaire que la longueur du texte ne soit pas excessive. Elle variera, compte tenu de la densité du propos, autour de 700 mots.

La règle sera de réduire le texte au quart environ de cette longueur. B.O., juiillet 1983 .

Les expressions que nous avons soulignées constituent les points clés. Le tableau qui suit les explicite et récapitule les impératifs du résumé.

\begin{tabular}{|l|l|}
\hline $\begin{array}{l}\text { 1. "Le résumé suit le fil du } \\
\text { développement. " }\end{array}$ & $\begin{array}{l}\text { - Il est impératif de respecter } \\
\text { l'ordre des idées. }\end{array}$ \\
\hline $\begin{array}{l}\text { 2. "Une version condensée } \\
\text { mais fidèle. " }\end{array}$ & $\begin{array}{l}\text { - La fidélité est le second impératif. } \\
\text { Le résumé ne change : } \\
- \text { ni l'organisation du texte } \\
\text { - ni son sens. }\end{array}$ \\
\hline $\begin{array}{l}\text { 3. "Il ne change pas le système } \\
\text { de l'énonciation. " }\end{array}$ & $\begin{array}{l}\text { - Si le texte est à la personne, le } \\
\text { résumé le sera également. }\end{array}$ \\
\hline $\begin{array}{l}\text { 4. " Il s'interdit le montage } \\
\text { de citations. " }\end{array}$ & $\begin{array}{l}\text { - Un résumé n'est pas une suite } \\
\text { de morceaux de phrases } \\
\text { empruntées au texte. }\end{array}$ \\
\hline $\begin{array}{l}\text { 5. "La règle sera de réduire } \\
\text { au 1/4 de la longueur du } \\
\text { texte initial. " }\end{array}$ & $\begin{array}{l}\text { - La longueur du texte se calcule } \\
\text { en nombre de mots. Le résumé } \\
\text { comportera le quart des mots du } \\
\text { texte. Une marge de 10\% en plus } \\
\text { ou en moins est autorisée. }\end{array}$ \\
\hline
\end{tabular}




\section{ANNEXE II}

\section{Typologie de la Contraction de Texte}

\begin{tabular}{|c|c|c|c|c|c|c|c|c|c|}
\hline & \multicolumn{2}{|c|}{ Contrainte de base } & \multicolumn{4}{|c|}{ Conception de Pinvariance informative } & \multicolumn{3}{|c|}{ Organisation de $\dagger$} \\
\hline & $\begin{array}{l}\text { Réduction du } \\
\text { signifiant }\end{array}$ & $\begin{array}{l}\text { Invarionce } \\
\text { informative }\end{array}$ & $\begin{array}{l}\text { Informotion } \\
\text { critique }\end{array}$ & $\begin{array}{l}\text { Sélection de } \\
\text { l'information }\end{array}$ & Thématisotion & $\begin{array}{l}\text { Distonce } \\
\text { énenciative }\end{array}$ & Linéarité & Citotions & $\begin{array}{l}\text { Reprise des } \\
\text { signifients }\end{array}$ \\
\hline Résumé scolaire & + & + & - & - & - & - & + & - & - \\
\hline Résumé informotif & + & + & - & - & - & -+ & -+ & - & + \\
\hline Résumé indicatif & + & + & - & - & + & -+ & + & - & + \\
\hline $\begin{array}{l}\text { Résumé des } \\
\text { conclusions }\end{array}$ & + & - & - & + & - & -+ & (1) & - & + \\
\hline Analyse & + & + & - & - & - & + & - & -+ & -+ \\
\hline Résumé critique & + & + & + & - & - & + & -+ & - & + \\
\hline Résumé synthétique & + & - & - & + & - & + & - & -+ & + \\
\hline Montoge & + & + & - & - & -+ & -+ & + & -+ & -+ \\
\hline Indexation & + & + & - & + & + & - & -+ & - & -+ \\
\hline
\end{tabular}

Le symbole " + " indique la présence, dans un type de texte contracté, de la caractéristique figurant dans la colonne correspondante. Le symbole " - " indique son absence, " - + " indiquent l'existence des deux possibilités. Pour la distance énonciative, " + " indique la distance maximum, " - " indique la distance nulle, "- + " indiquent l'existence des deux possibilités.

(1) La notion de linéarité ne s'applique pas ici.

POUZET, R. (1981). «La contraction de texte - typologie ». Condenser 2 (1981) : 5-18. 


\section{ANNEXE III}

Description des cours de réduction de textes offerts dans les écoles de traduction au Canada

Collège universitaire de Saint-Boniface, University of Manitoba, Winnipeg, MB 1er cycle - $2 e$ année - Obl. - 122-210 Analyse et résumés de textes I (3 cr.)

Apprentissage des techniques d'analyse et de résumé de textes pragmatiques français de façon à développer l'aptitude interprétative (extraction du sens) et les capacités expressives (reformulation) chez l'étudiant ou l'étudiante. Les textes, écrits ou audiovisuels, touchent à l'actualité et aux divers domaines avec lesquels le traducteur sera appelé à se familiariser.

1er cycle - 3e année - Obl. - 122-310 Analyse et résumé de textes II (3 cr.)

Pratiques des techniques d'analyse et de résumé à partir de textes français ou anglais. La reformulation en français sera l'occasion d'un exercice intellectuel proche de la traduction. Les recherches documentaires viseront non seulement à l'amélioration des connaissances générales mais aussi à l'établissement de glossaires.

\section{Université Laurentienne, Sudbury, ON}

1er cycle-3e année-Opt.-TRAN 3146F Contraction de textes et comptes rendus $(3 \mathrm{cr}$.)

Ce cours présente une introduction à la pratique de la contraction de textes. Dans l'exercice de la profession, les traducteurs sont souvent désignés comme traducteurs-rédacteurs de comptes rendus : ils doivent pouvoir assurer concurremment ces deux fonctions. Rédaction de comptes rendus analytiques ou in extenso.

\section{Concordia University, Montréal, QC} 2e cycle - Obl. - TFRA 524 Contraction de textes $(3 \mathrm{cr}$.)

(1) Se familiariser avec les divers résumés documentaires à finalité pédagogique et professionnelle : résumés-condensés, résumés-sommaires et résumés-synthèses. 
(2) Développer ses aptitudes interprétatives (analyse) et ses capacités expressives (synthèse) par la rédaction de divers types de résumés documentaires.

(3) S'initier aux langues de spécialité par des recherches documentaires et l'établissement d'un dossier-sujet.

(Louise-L. Larivière)

McGill University, Montréal, QC

672-23o Précis-writing/Contraction de textes (3 cr.)

This course is intended to give translators additional training which will be of practical use in their work. The emphasis will be on the understanding and analysis of texts in order to determine the essential elements needed in precis-writing. Exercises based on varied French texts are intended to familiarize students with the preparation of texts of a practical nature, e.g. reports, minutes, abstracts. The objective of the course is to train students to summarize a given text accurately and concisely in a way that respects the form and sophistication of the original text and exploits the idiomatic nature of the language.

Ce cours vise à donner au traducteur une formation complémentaire utile à son travail. L'accent sera mis sur la compréhension et l'analyse du texte pour en dégager les éléments essentiels à partir desquels l'étudiant aura à rédiger un résumé. Exercices à partir de textes anglais variés visant à donner à l'étudiant l'habitude de rédiger des textes d'ordre pratique : comptes rendus, procès-verbaux, condensés. L'objectif sera d'arriver à une expression juste et concise respectant la forme et le niveau du texte, la correction et le caractère idiomatique de la langue.

\section{Université Laval, Québec, QC}

\section{Opt. FRN-17265 Synthèse de documents}

Optionnel dans le bloc langue et culture. Objectif : apprendre à traiter un document, de l'écrit court au dossier plus volumineux, en vue de le réduire en un texte synthétique, fidèle et compréhensible. Développer 
son esprit d'analyse et de synthèse. Contenu : pratique de la contraction de textes ; analyse de textes synthétisés ; rédaction de résumés et de synthèses diverses.

\section{COMMENTAIRE}

À Saint-Boniface, on ne précise pas quels types de résumés sont enseignés, mais on indique que, dans le cours de $2^{\mathrm{e}}$ année, les résumés sont faits à partir de documents de nature pragmatique, écrits ou audiovisuels, qui touchent à l'actualité et à divers domaines avec lesquels les langagistes seront appelés à se familiariser, tandis que dans le cours de $3^{\text {e }}$ année, les documents de départ sont soit en anglais, soit en français pour aboutir à un résumé en français. Ả Laurentienne, on enseigne les résumés de type «contraction de texte» et les comptes rendus. À McGill, on mise sur le transfert linguistique puisque dans le cours en anglais, on verra à traduire des textes en français et, dans le cours en français, des textes en anglais. Dans les deux cas, les textes sont de nature pratique. A Laval, on enseigne la contraction de texte et des résumés et synthèses diverses. A Concordia, lorsque le cours sur le résumé se donnait, il ne comportait pas de description dans l'annuaire (ce cours a été aboli postérieurement à la rédaction de cet article à l'automne 1999) ; aussi, comme nous enseignions ce cours, nous y avons inscrit la description qui apparaissait dans notre plan de cours. Même si le cours s'intitulait "contraction de texte», nous enseignions trois types de résumés : les condensés (dont la contraction de texte), les sommaires (dont le résumé analytique) et les synthèses (dont la synthèse documentaire). Lorsque le cours de résumé se donnait à l'Université de Montréal, il s'intitulait aussi «contraction de texte».

En général, ce sont les résumés de type contraction de texte qui prévalent dans ces institutions. Rappelons que la contraction de texte, telle qu'elle a été développée en France, est un résumé de type scolaire qui consiste en une réduction à l'échelle du texte de départ, qui constitue, bien souvent, une épreuve à des concours d'entrée ou à l'obtention d'un diplôme et qui est régi par des normes. Par conséquent, les ouvrages sur les résumés, conçus pour préparer les élèves à satisfaire à ces normes, adoptent presque tous la même démarche : définition du résumé, explication des normes, méthodes à suivre, exercices pratiques (de généralisation), résumés commentés, textes d'application avec corrigés, textes d'entraînement regroupés par sujets, liste des sujets donnés à des concours antérieurs. 
Indépendemment des normes françaises, la contraction de texte s'apparente à un texte traduit en ce sens que, tout comme lui :1) il est un document second reproduisant, sous une forme définie, un document primaire;2) il rend compte de la totalité du contenu du document primaire ;3) il est soumis à des phases de décodage et d'encodage qui s'effectuent au moyen de techniques d'analyse et de synthèse ;4) il reproduit le style d'écriture du texte de départ. C'est sans doute à cause de sa ressemblance avec le texte traduit que le résumé de type contraction de texte est si largement enseigné.

L'enseignement de ce seul résumé est-il toutefois suffisant? Tout dépend des raisons pour lesquelles on l'enseigne. À Saint-Boniface et à Concordia, on vise à développer les aptitudes interprétatives (analyse) et les capacités expressives (synthèse) chez les étudiants et les étudiantes ; à Laurentienne, on vise à préparer les futurs langagistes à leurs tâches de rédacteurs-traducteurs ou de rédactrices-traductrices de comptes rendus. À Concordia, on vise de plus à familiariser la population étudiante avec les divers types de résumés et à l'initier aux langues de spécialité par des recherches documentaires et par l'établissement d'un dossier-sujet. À McGill, on vise à donner aux langagistes une formation complémentaire à leur travail pour en arriver à une expression juste et concise respectant la forme et le niveau du texte, la correction et le caractère idiomatique de la langue. À Laval, on vise à développer l'esprit d'analyse et de synthèse.

Si le but visé est de développer l'esprit d'analyse et de synthèse, la contraction de texte est un moyen efficace parmi d'autres. Toutefois, comme nous l'avons vu, dans notre exposé, le résumé scolaire n'est pas un véritable document produit conformément à une démarche propre à la communication. Aussi, il faut maintenir des cours de résumés distincts dans les écoles de traduction. Montréal a fait disparaître le sien et Concordia aussi. On a considéré que les cours de résumé faisaient double emploi avec les cours de rédaction (générale ou professionnelle). On n'a pas compris que le résumé constitue un genre en soi, ayant une finalité et des caractéristiques propres, et non pas un simple moyen d'améliorer sa façon d'écrire. De plus, le résumé est en mesure de satisfaire aux exigences des cours de transfert puisqu'il est toujours possible de résumer dans une langue autre que celle du texte de départ. 


\section{RÉFÉRENCES}

AFNOR (1984). Documentation : Recommandations aux auteurs des articles scientifiques et techniques pour la rédaction des résumés. Norme française homologuée $Z$ 44-004. Paris : Association française de normalisation.

Arambourou, Ch., Texier, F. et F. Vanoye (1991). Guide du résumé de texte. Paris : Hachette éducation. (Faire le point. Méthode). Publié antérieurement sous le titre :Guide de la contraction de texte.

Beaudet, Céline (1994). «Pour une typologie des résumés fonctionnels : réflexion sur l'acte de classification". Technostyle, 11(3-4), 49-58.

Bernié, Jean-Paul (1993). Raisonner pour résumer. Une approche systémique du texte. Berne : Peter Lang. (Sciences pour la communication 37).

Blicq, Ron S. (1992). Technically-write! communicating in a technological era. Canadian fourth edition. Scarborough (ON) : Prentice-Hall Canada Inc.

Carloni, J. C. (1984). Pratique de la contraction de texte aux concours des grandes écoles. Montreuil : Bréal.

Fournier, Jacques et Roger Dutertre (1993). Le résumé de textes par l'exemple : promotion sociale, baccalauréat, concours administratifs, grandes écoles... Paris : Roudil. (Les études par l'exemple).

Giquel, Françoise (1990). Réussir le résumé de texte. Paris : Editions d'organisation. (Méthod'Sup).

Guinchat, Claire et Michel Menou (1980). Introduction générale aux sciences et techniques de l'information et de la communication. $2^{\mathrm{e}}$ éd. rev. et augm. par Marie-France Blanquet. Paris : Les Presses de l'Unesco.

ISO (1976). Documentation - Analyse pour les publications et la documentation. $1^{\mathrm{re}}$ édition. ISO 214-1976(F). Genève : Organisation internationale de normalisation.

ISO (1981). Information et documentation - Vocabulaire - Chapitre 3a) : Acquisition, identification et analyse des documents et des données. ISO 5127/3a)-1981 (E/F). Genève : Organisation internationale de normalisation.

Jewinski, Ed. et Judi Jewinski (1992). Savoir préparer un résumé : méthode pour les gestionnaires. Ottawa : Presses de l'Université d'Ottawa. Traduction de How to write an executive summary. 
Landau, Thomas (ed) (1966). Encyclopaedia of librarianship. 3rd ed. London : Bowes and Bowes.

Larivière, Louise (1989). «Le résumé documentaire : problèmes et solutions». Technostyle 8(1-2), 1-13.

Larose, Robert (1992). La rédaction de rapports : structure des textes et stratégie de communication. Québec : Presses de l'Université du Québec.

Maury, Pierre (1982). Résumer les textes : la synthèse, la nuance, le style Verniers, Belgique : Nouvelles éditions Marabout. (Marabout Flash 459).

Meney, Lionel (1993). «Un exercice de rédaction : la contraction de texte». Technostyle 11(2), 18-38.

Moreau, Jean A. (1984). La contraction et la synthèse de textes. Paris : Nathan. (Nathan université : Littérature française).

Pouzet, R. (1981). "La contraction de texte - typologie». Condenser 2, 5-18.

Robinson, Patricia A. (1985). Fundamentals of technical writing. Boston : Houghton Mifflin Company.

Russell, Pamela (1994). «Investigating summary typology : considerations for classification». Technostyle 11(3-4), 37-47.

Sabbah, Hélène (1991). Le résumé (1) : initiation : définitions, méthode, exercices, mots clés, corrigés. Paris : Hatier. (Les Méthodiques).

SOQUIJ (1993). Droit du travail express. Montréal : Société québécoise d'information juridique.

Stalloni, Yves (1990). Le résumé au bac. Paris : Bordas.

Valentine, Egan (1990). Précis-writing : initiation à la contraction de texte Montréal : Sodilis.

Université Laval. Bibliothèque (1983). Répertoire de vedettes-matière. $9^{\mathrm{e}}$ éd. Québec : La bibliothèque. 